\title{
Individualized Reaction Movements For Virtual Humans
}

\author{
Alejandra García Rojas M.* \\ EPFL-VRLab
}

\author{
Frédéric Vexo ${ }^{\dagger}$ \\ EPFL-VRLab
}

\author{
Daniel Thalmann \\ EPFL-VRLab
}

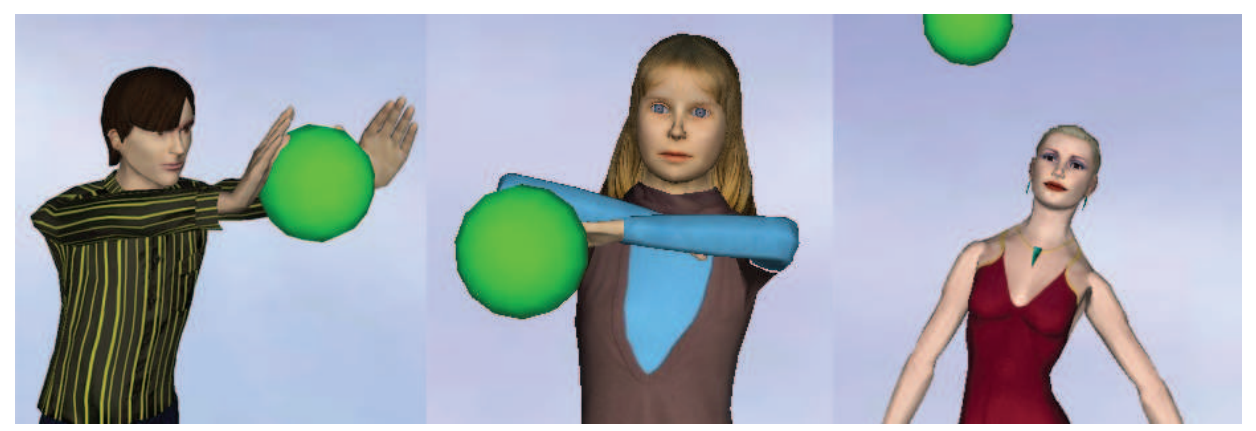

Figure 1: Individual people: different reactions.

\begin{abstract}
Virtual Humans creation aims to provide virtual characters with realistic behavior, which implies endowing them with autonomy in an inhabited virtual environment. Autonomous behavior consists in interacting with users or the environment and reacting to stimulus or events. Reactions are unconscious behaviors which are not often implemented in virtual humans. Frequently, virtual humans show repetitive and robotic movements which tend to decrease realism.

To improve believability in virtual humans we need to provide individuality. Individualization is achieved by using human characteristics like personality, gender, emotions, etc. In this paper, we propose to use those individual descriptors to synthesize different kinds of reactions. We aim that individualized virtual humans react in a different way to the same stimuli. This approach is achieved by observing real people reacting. Thanks to those observations, we stereotyped reactive movements that can be described by individual characteristics. We use inverse kinematics techniques to synthesize the movements. This allows us to change reaction movements according to the characteristics of the stimuli and to the individuality of a character.
\end{abstract}

Keywords: virtual humans animation, reaction movement, semantic model

\section{Introduction}

A realistic Virtual Human behavior lies in a good simulation of its autonomy. There exist models of behavior that provides autonomous cognitive control[Doyle 2002], and a good perception

\footnotetext{
*e-mail:alejandra.garciarojas@epfl.ch

†e-mail:frederic.vexo@epfl.ch

†e-mail:daniel.thalmann@epfl.ch
}

of the environment [Conde and Thalmann 2005]. This autonomy consists in self awareness and task performance. However, we believe that not predefined movements, like reactions, can increase the realism in virtual characters.

Human's reactions to unexpected events vary from one person to another. To achieve a good simulation of reactions we need to address several aspects of the process. The better way to understand and analyze this process is through observation. In this paper, we present an observation-based approach to stereotype reaction movements of the humans. This kind of approach has already been used to provide autonomous behavior to virtual humans [Badler et al. 1999].

We are interested to model the kind of reactions that require short time of response. As a consequence, our observation case study consists in a simple reaction movement: make people react to a ball coming towards them. Reactions to this kind of situations vary in many different ways from one person to another; they also vary over time, it means that the same person will react differently to the same stimuli. This variation depends on the personality and internal state of each person at that moment.

The results of the analysis of the case study allowed us to stereotype this reactive example. The groups of movements identified, could be semantically described in terms of individual parameters such as gender, personality, emotion etc. Therefore, we focussed on synthesizing and parameterizing the defined kind of movements, in order to be able to produce variations of the same movement. The parameters of a kind of reaction could be arbitrary set according to individual descriptors. We have to remark that in this paper, we do not aim to predict the kind of reaction of a kind of person, but to provide different kinds of movements according to the assumption of individualities.

To reproduce the stereotyped movements, we used inverse kinematics. This technique allows changing some movement settings according to individual parameters. We can also change movements according to the characteristics of the stimuli, such as direction, size or velocity.

The way that inverse kinematics, individual parameters and reactive behavior are connected is presented in a semantic diagram (ontology). Based on this context, we built a system where virtual humans are able to produce a spontaneous behavior according to their internal information and external stimuli coming from the virtual 


\section{environment.}

The content of this paper is organized as follow: in section 2, we present the related work about reactive motions. Then, in section 3 , we describe the case study of real people reacting. In section 4 , we give a semantic representation of individual reaction behavior for virtual humans, followed by the synthesis of these reactions using inverse kinematics in section 5. Finally, in section 6 we present our conclusions and future work.

\section{Related work}

In game industry, reactive animations to stimuli are made using motion captured sequences. This is commonly found in battle or sport video games. The use of motion capture is useful because reaction movements are relatively limited to a group of movements. To be able to produce reactions, we need a large database of motion captured movements and search for the most adequate movement(s). Afterwards we can apply motion blending, dynamic constraints [Zordan and Hodgins 2002] or physical constraints [Tang et al. 2006] to make it the most realistic possible. Komura et al. [Komura et al. 2005] used motion capture mixed with inverse kinematic momentum-based. IK was used to keep the balance of the character.

In the above approaches, authors are dealing with the passive effect of an impact. This means that the character has been hit and they compute how he will fall down in a realistic way. In [Faloutsos et al. 2001], this kind of work is extended by making the arms of the character to react in a protective way when he is falling. We believe that reaction movements in virtual characters give the impression of being alive; except for the fact that there is any individualization of reactive movements for each character.

The simplest spontaneous behavior in human beings produced by external stimuli is the reflex. Reflexes are movements not controlled by consciousness and they are regulated by the nervous system. They can be considered as the basis of movements or even the root of human movement. Reflex movements were already explored in [Gutierrez et al. 2004]. They got inspired on the nervous system and used inverse kinematics for the implementation. They made virtual humans able to react spontaneously to stimuli. Even their implementation does not consider individual reactions, it is possible to discuss until what level people can react the same way.

In this paper we present an experiment to analyze how different can be people's reactions. This is presented in the next section.

\section{Study Case}

We can consider reactions produced by reflexes as primitive responses that protect our body from danger and help us to adjust to our surroundings. The movement performed during a reaction depends on many factors. The most evident factors are the physical characteristics: gender, age, body constitution, etc. e.g. aged people have uncertain or slow reactions. Other less evident factors, are the internal or psychological parameters of the person: personality, emotional state, etc. e.g. considering a person who has been frightened; if she is highly neurotic she might shout out very loudly as a reactive response.

A common way to model human behaviors is by means of the observation and analysis. We have made an experiment in which we make people react to an stimuli: a ball thrown towards them. We annotated their morphology to be able to make future classifications. Our sample was of 20 people of different gender, nationality and age. With a hidden camera we record the subjects; while they were answering a questionnaire, suddenly we drop a ball towards them. Some screen shots of videos are presented in the figure 2.

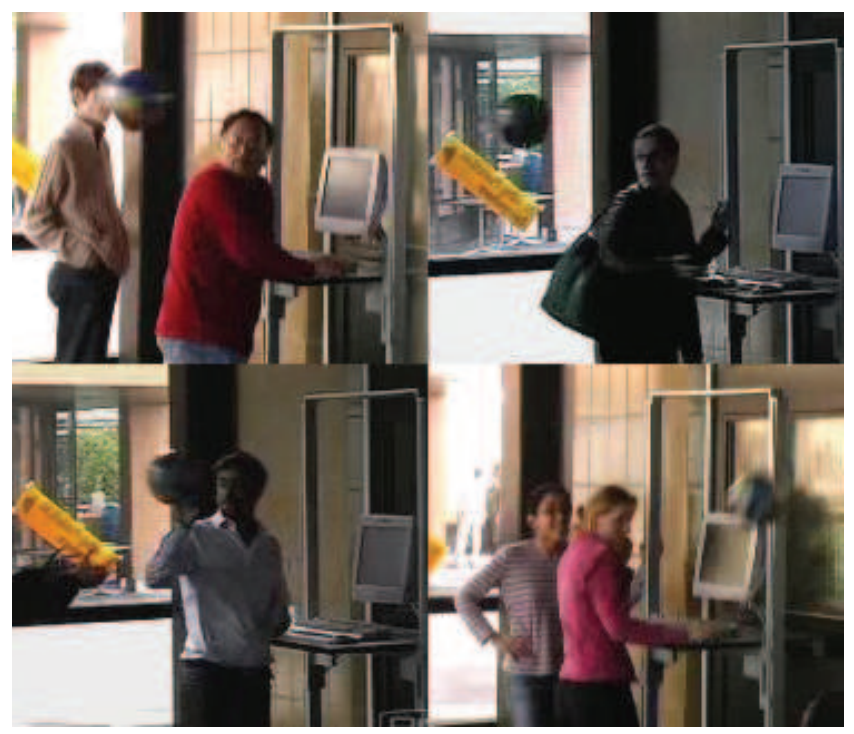

Figure 2: Videos of the study case of people reacting.

During the analysis of data and videos, we have notice that some subjects do not move too much at the time of reacting, others avoid the ball, others protect themselves, and so on. The results of this analysis was the identification of common characteristics in the reactive movements. We could distinguish 3 types of reactions: intersect, avoid, and protect. They are briefly described hereinafter:

Intersect to avoid danger by putting something in between, in this case the hands.

Avoid to move the body in the opposite direction where is the stimuli coming from.

Protect to cover a part of the body to avoid the impact (e.g. use the hands to protect the face).

The observed types of reactions performed by the people were indeed with different intensities or even mixed. To be able to make a synthesis those reactions, we needed to generalize some types of movements for its classification. This generalization is, for example, the stress reaction or closing the eyes are intent of avoidance.

We consider that the differences in reactions came out because of two factors: external and internal parameters. In this case, the external parameters are the direction and velocity of the stimuli. Internal parameters are comprised in the individuality of the person. Therefore, according to the external information and internal state of a person (individuality), an evaluation process takes place and takes the decision of the movement to perform.

The sample we took for this test is too small to allow us to highlight relations between individual parameters and kinds of reactions. We may need to address some psychological studies to be able to establish a relationship. In this paper, we are not interested in providing a kind of reaction according to a given parameters, but in generating different kinds of reactions that can be parameterized according to people's individuality.

Based on the knowledge acquired in this experiment, we can synthesize the kind of movements and parameterize them according to individual descriptors. For this, we made a semantic representation 
of a reactive behavior that considers the mentioned external and internal factors; this is presented in next section.

\section{Semantic Representation of Individual Reactions}

Semantic representations are usually distinguished by the use of ontologies, it aims at the specification of a conceptualization [Gruber 1991]. The exploitation of ontologies is present in many knowledge domains. In computer graphics it has been explored from shape analysis[Leon et al. 2005] until gaming analysis[Zagal et al. 2005] passing by motion synthesis[Garcia-Rojas et al. 2006].

Creation process of virtual humans was presented in [Garcia-Rojas et al. 2005]. It gives a step towards in the virtual human's 3D graphic representation by providing a semantic layer to synthesize its geometry. Virtual Humans, as part of virtual environment applications can be formally represented not only as a 3D shape, but also as a dynamic entity with multiple visual representations and functionalities [Gutiérrez et al. 2005]. This allows for dynamically scaling and adapting the object's geometry and functions to different scenarios. The same structure can be applied to model behaviors, where we need scalability and adaptability to implement and test models.

To build an ontology, we start from finding concepts and relations among them. In our representation, we have a virtual human that is attributed with the following features:

- Morphological Descriptor: describes the shape of the human body as human being; it has the properties weight, height, gender, age, etc.

- Individuality: describes parameters that can define the individuality of a person, like personality, emotional state, cultural background, etc.

- Body: For a virtual human the body is constituted by a geometry and an a skeletal structure that is used to drive the animation. For our application we use H-Anim to describe the body.

- Behavior Controllers: they are algorithms used to produce behavioral animation. The class specifies the inputs required for the algorithm to work and the outputs (usually animation sequences or specific joint values) it is capable to produce.

- Reaction behavior: algorithm that simulates the evaluation process where, considering the virtual human attributes and the nature of an stimuli, it will generate a kind of reaction.

- Inverse Kinematics: algorithms that provides a skeletal posture defined by specifying a target location to end-effectors. The joint angles of the control skeleton are computed so that the end-effectors reach their targets as close as possible.

Those concepts describe the virtual human in a general way. They are taken into account at the time to produce an individualized reaction.

Other concept we include in our representation, that is part of the virtual environment, is the Stimuli. The stimuli has properties by itself, such as position and orientation; and if it is moving, velocity and direction; and a level of danger. Those properties will influence the reaction of the virtual human.

In the figure 3, we present an ontology diagram to model virtual human features for an individual reactive behavior. Concepts are connected by arrows. These connections represent the properties of concepts; for example a virtual human has geometry, morphology description, individuality, etc.

General concepts can inherit to specific ones; they are connected by dashed lines. Reaction and Inverse Kinematics are subclasses of Behavioral Controller (here, subclasses behave like in object oriented paradigm). We consider a Behavioral Controller those algorithms that have inputs, make an evaluation according to the internal state of a virtual human and convey results in outputs. For the Reaction controller, the inputs are: stimuli, morphological and individual descriptors; and the output are the inverse kinematics constraints. This controller is an evaluation algorithm that defines the kind of movement to perform according to the inputs. And it will generate the end-effectors position and orientation used by the inverse kinematics controller.

We can see that we have different levels of concepts that are children of behavior controller. One's output serves as input of the other. Under this scheme, for behavior, emotion and personality models, it is possible to make their implementation as it better fits.

In the next section, we present the implementation of the above concepts in the application test.

\section{Individual Reaction Simulation}

The implementation of the individual reactions is composed of two parts divided in subsections: how we implemented the movement, this means the inverse kinematics; and the behavior controller of reaction which considers the individual traits.

\subsection{IK-based Movements}

The Inverse Kinematics library we used has been developed by [Baerlocher and Boulic 2004]. This tool allows controlling more than one end-effector at the same time. It is possible to associate a distinct priority level to each effector to guarantee that the most important goals are achieved first. It also allows setting an engagement of the end effector joint with its parent joints in different levels. Moreover, we can treat the center of mass of the body as an end-effector to ensure the static equilibrium of a figure [Boulic et al. 1996]. Combining effectors and their associated weight and priority levels we can simulate complex and believable synergistic postures.

Using this library, we created three inverse kinematic end-effectors to synthesize reactions: right arm, left arm and the spine until the skull base. Through these effectors we are able to set actions at joint level to give end-effector position and /or orientation. For example, for the intersect movement, we set the position of the wrist joint to the position where the stimuli will be at the moment of the intersection.

As we mentioned, more than one end-effector can be set, thus we can set more than one position and orientation constraint on one arm with different weight and priority levels depending on the kind of pose we want to produce. This priority level goes form 1 to 10 where 10 is the maximum. For example, in the protection motion the orientation of the low part of the arm is important, we can have the palm of the hands covering the face, but we can also have it in the opposite direction. There is a different meaning of the movement in each of those positions; covering the face could mean that the person is afraid, while the other one could be a defensive action.

Within this inverse kinematics library, we can assign different levels of joint recruitment. This level goes from 1 to 10 , where 10 is the 


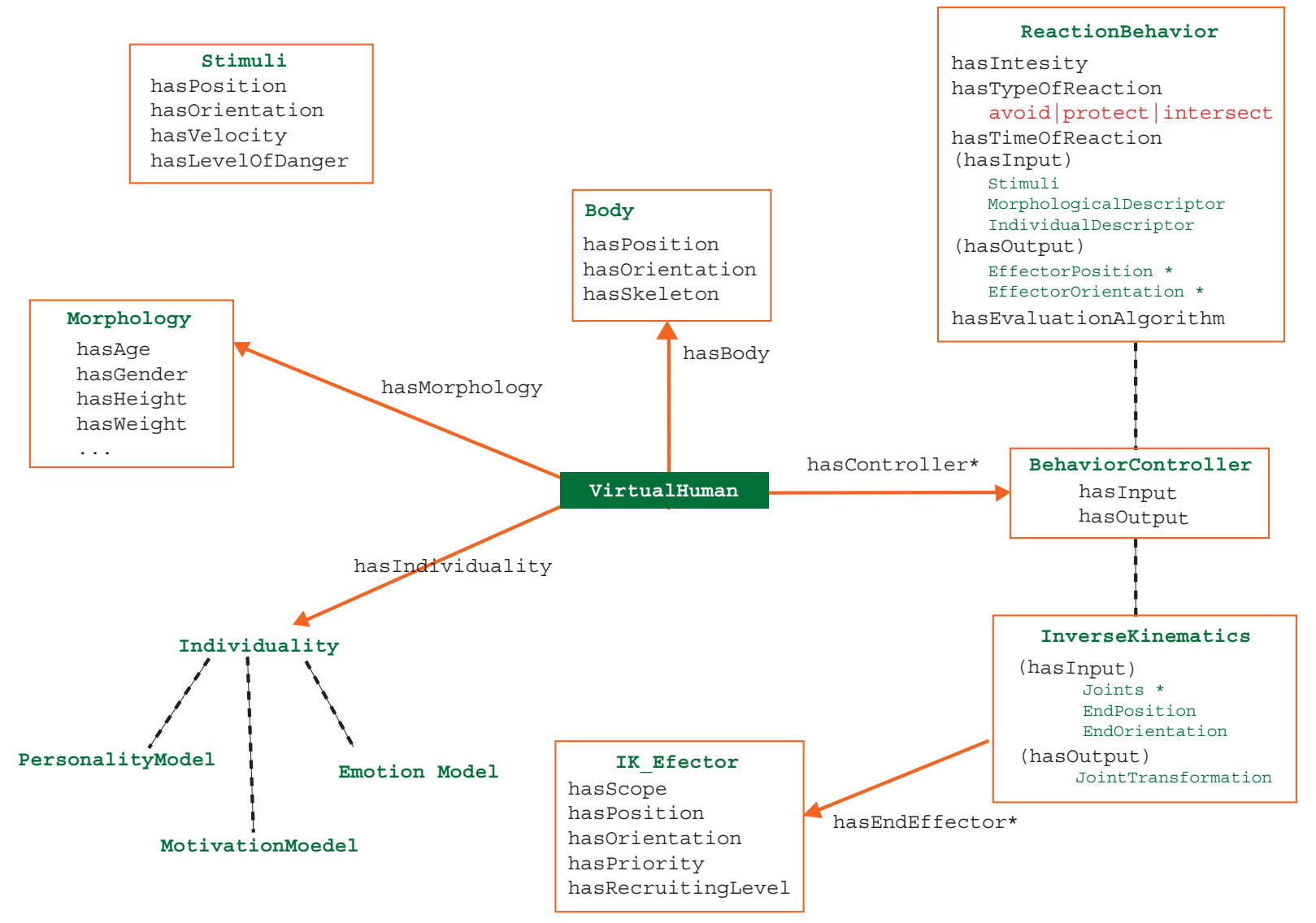

Figure 3: Semantic representation of individual reaction modeling. 
recruitment of all joints assigned in the IK controller. This property is useful to define the quantity of motion we want to propagate in the body. For example in the avoidance motion we can move from the head until until the entire torso. These movements may depend on the level of danger or the direction of the stimuli: if the level of danger of the stimuli is medium the movement will be propagated in all the entire torso, if it is high the character may step backwards.

In summary, the Inverse kinematics implementation allows us to set more than one end-effector at time. Using different levels of priorities, we define the importance of the goals to achieve. To define amount of joints that will be involve to achieve a goal, we can set a level of recruiting.

The different end-effectors constraints with their properties are defined in the behavior algorithm. In the next subsection we will explain a simple behavior algorithm and the implementation of each kind of reaction identified in our case study.

\subsection{Synthesis of Reactive Motions}

This test application is implemented using the VHD++ framework described in [Ponder et al. 2003]. In this development tool, we can load characters with H-Anim structure, and create the described inverse kinematics controllers for the arms and the spine. VHD++ architecture provides a high-level control of the environment through python script modules. Using this interface we can easily define higher level behaviors to lower level components.

The algorithm of reaction behavior for this demonstrative application is quite simple. We observed different kind of behaviors in our case study, and we have hypothesized different kind of reactions to our individualized virtual humans. This test considers three different characters: Brian, Lydia and Johanna. We have defined individual descriptors for each one inside the ontology. These instances are presented in the figure 4 . The morphology descriptor refers to the external physical aspect of the character. The personality definition is the five factor model [McCrae and John 1992], which is on one of the most popular computational models of personality. The emotion is only defined by an emotional state assigned to the character at the moment of the reaction.

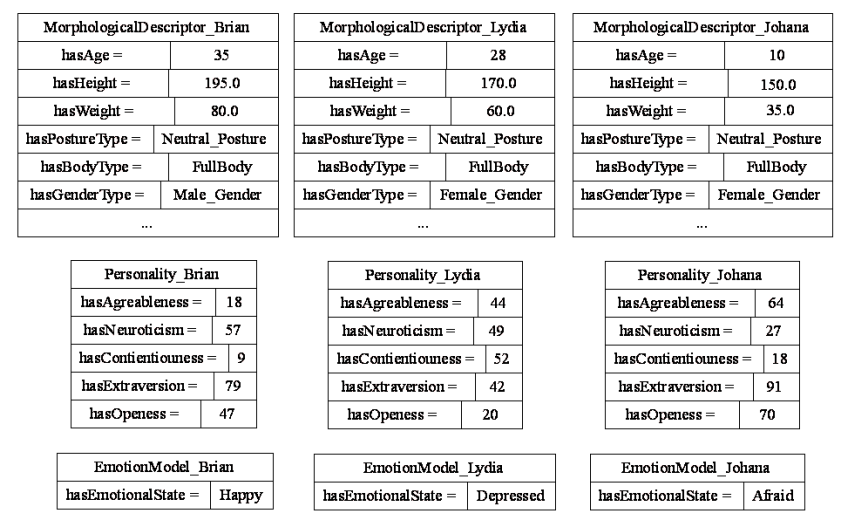

Figure 4: Individuality definition of virtual humans.

We have also defined properties of the stimuli, which is in this case a ball coming towards the character. Its properties are direction and velocity, which are initial parameters for dropping it; level of danger, which is set as medium (not dangerous); and size equals to $13 \mathrm{~cm}$ of radius.
The inputs of the algorithm are the individual descriptors and the properties of the stimuli. Therefore, the reactive behavioral algorithm chooses which kind of behavior the character will perform. This algorithm is something like:

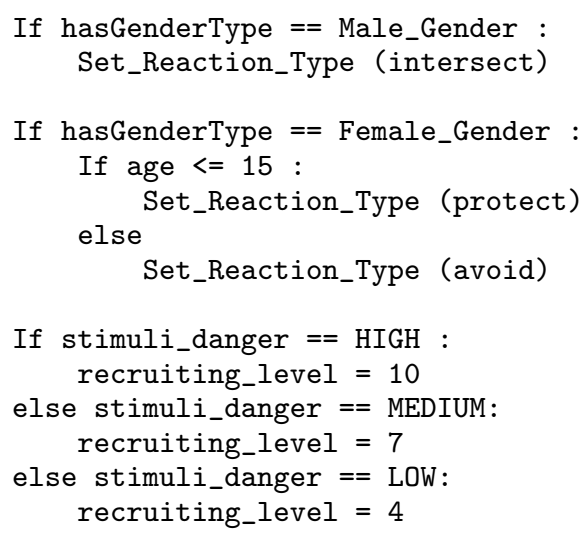

The first part of the algorithm chooses a movement according to the gender and age. The second part defines the recruiting level for the joints according to level of danger of the stimuli. In our implementation, the recruiting is applied to the main inverse kinematics controller of the selected movement. This means that for the interactive and protection it will be applied to the arms, and for avoidance to the spine end-effector.

We now define individualized constraints depending on the reaction to send to our inverse kinematics library. In the intersection reactive movement, performed by Brian, we compute the vector position for the end effectors of each hand. This position is the position of the stimuli at the moment when it is reachable by the virtual human arms, plus the separation of the arms according to the size of the stimuli.

To give the impression of a more natural movement, we compute an orientation effector of the wrist to flex it perpendicular to the direction of the ball; this orientation constraint is set with a lower priority level and with a recruiting level of 0 because it only involves wrist joint. Some frames of this reactive movement are presented in the figure 5 .

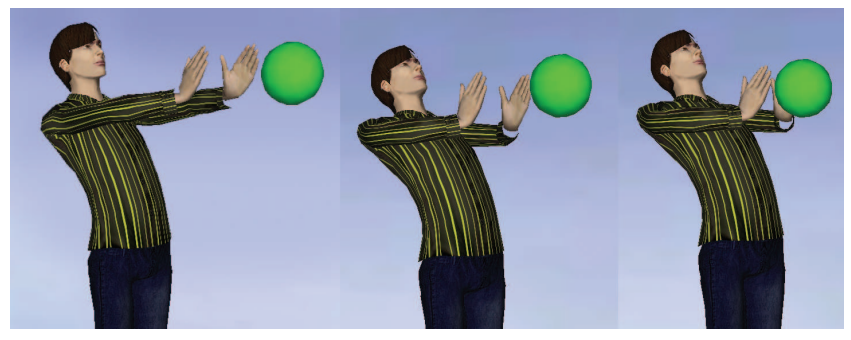

Figure 5: Frames for intersect movement.

Avoid movement of Lydia, is function of the direction and level of danger of the stimuli. We compute the direction vector of the stimuli to get the direction of the movement which is perpendicular of the stimuli direction. The magnitude of the movement is function of the size of the stimuli. This movement is applied to the spine end-effector. We can also add some extra movements to the arms to be more natural. We define a new position to the arms end-effectors with a lower level of priority and recruiting.

set IK_spine_position = vector_dir $*$ danger

Being able to set other kind of movements in the body, like the arms 
movement in the case of avoidance, can be considered as an advantage; because it allow us to provide a different intensity or impact in the movements and express better an emotion or personality. Some key frames of the avoid movement are presented in the figure 6 .

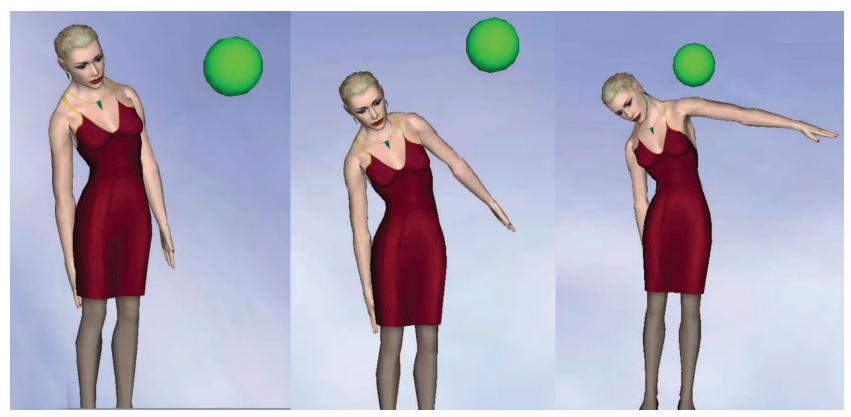

Figure 6: Frames for avoidance movement.

Protection movement performed by Johanna was more complicated to synthesize, but we could produce numerous possible movements. For this movement we raise the elbows until the lowest part of the stimuli and raise the wrists at different heights, higher than the elbows. Here, we have many choices at the time of positioning and orienting the wrists. An example of different movement options is defined in the following algorithm:

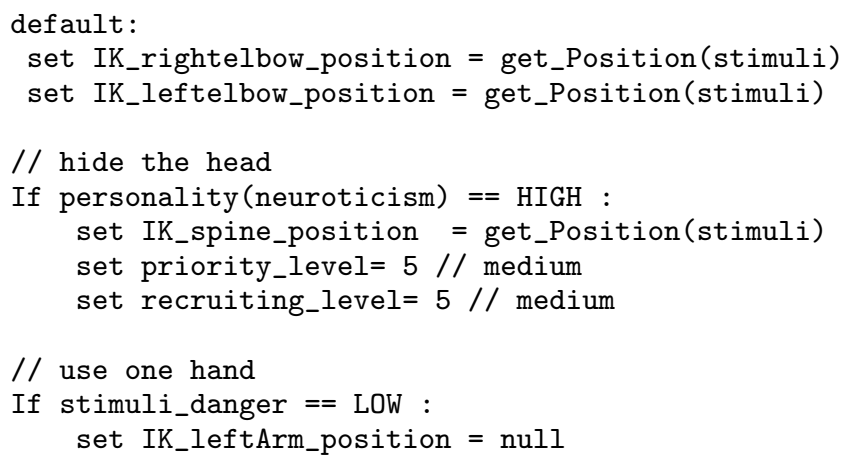

In the three cases presented in the figure 7, we could see that protective movement can contain interactive and avoidance movement, but the way it is performed is more complex, and as a consequence, more constrained. Therefore we cannot define it only as a mix of those movements.

We have shown that, once the kind of behavior is selected, we use the information of the stimuli parameters to compute the basic pose that the character may take. After that, we use character's individual traits and properties of the stimuli to set different parameters to the inputs of inverse kinematics to vary movements. We have also to add other parameters as the velocity of the movement, which depends on the time elapsed before reaction and on the level of danger of the stimuli.

\section{Conclusion and Future Work}

In this paper, we have presented how we synthesize different kinds of reactive movements according to individual character descriptors. Based on an observation experiment made with the participation of real people, we have defined three kinds of reactive movements. To synthesize the movements identified, we stereotype each

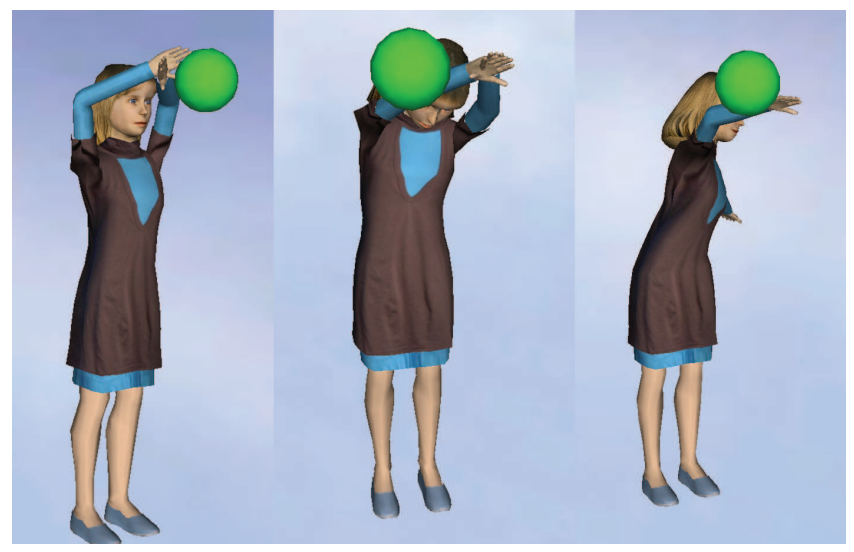

Figure 7: Different kinds of protective movements according to behavior parameters.

one to $\mathrm{b}$ able to reproduce them using Inverse Kinematics. Using this technique we could variate the movements by changing parameters like priority of end-effector performance, recruitment of joints per end effector, and end-effectors position and orientation.

In the test application presented, we can model individualized kinds of reactions. The individualization takes place in a reaction behavior algorithm, which is quite simple in our example. This algorithm showed how individual descriptors and the properties of a stimuli were translated as inputs to the inverse kinematics. We did not want to make more complex algorithm because it implies some knowledge in psychology. However, our semantic representation allows implementing easily any model of behavior.

For the future work, we want to define how we can mix the types of reactions reaction found. We will also establish the main inputs to the inverse kinematics in reactive movements. Moreover, we want to test different kinds of stimuli in a more complete scenario, like a reactive game.

\section{Acknowledgements}

This research has been partially funded by the Swiss Federal Office for Education and Science in the framework of the European Network of Excellence IST - AIM@SHAPE (http://www.aim-atshape.net).

\section{References}

BAdler, N. I., Chi, D. M., AND ChOpRA-Khullar, S. 1999. Virtual human animation based on movement observation and cognitive behavior models. In $C A, 128-137$.

BAERlocher, P., AND BOUliC, R. 1998. Task-priority formulations for the kinematic control of highly redundant articulated structures. In Proceedings of IEEE Conference Intelligent Robots and Systems IROS 98, 323-329.

BAERlocher, P., AND Boulic, R. 2000. Kinematic control of the mass properties of redundant articulated bodies. In Proceedings of IEEE Conference on Robotics and Automation, ICRA 2000, 2557-2562. 
BAERlOCHER, P., AND BOUliC, R. 2004. An inverse kinematics architecture enforcing an arbitrary number of strict priority levels. Vis. Comput. 20, 6, 402-417.

Boulic, R., MAs, R., AND Thalmann, D. 1996. A robust approach for the control of the center of mass with inverse kinetics. Computers and Graphics 20, Issue 5, Mobile Computing (September-October), 693-701.

Conde, T., And Thalmann, D. 2005. Autonomous virtual agents learning a cognitive model and evolving. In IVA, 88-98.

Doyle, P. 2002. Believability through context using "knowledge in the world" to create intelligent characters. In $A A M A S$ '02: Proceedings of the first international joint conference on Autonomous agents and multiagent systems, ACM Press, New York, NY, USA, 342-349.

Faloutsos, P., Van de Panne, M., and Terzopoulos, D. 2001. The virtual stuntman: dynamic characters with a repertoire of autonomous motor skills. Computers and Graphics 25, 6, 933-953.

Garcia-Rojas, A., Thalmann, D., Vexo, F., Moccozet, L., Magnenat-Thalmann, N., Mortara, M.; SpagnUOLO, M., AND GUTIERREZ, M. 2005. An ontology of virtual humans: incorporating semantics into human shapes. In Integration of Knowledge, Semantics and Digital Media Technology. The 2nd European Workshop on the (Ref. No. 2005/11099)., 714.

Garcia-Rojas, A., Vexo, F., Thalmann, D., Raouzaiou, A., K.Karpouzis, Kollias, S., Moccozet, L., AND Magnenat-Thalmann., N. 2006. Emotional face expression profiles supported by virtual human ontology. In Computer Animation and Virtual Worlds Journal, J. Wiley and L. Sons, Eds., vol. 17, 259-269.

GRUBER, T. R. 1991. The role of a common ontology in achieving sharable, reusable knowledge bases. In Proceedings of the Second International Conference on Principles of Knowledge Representation and Reasoning, 601-602.

Gutierrez, M., Vexo, F., And Thalmann, D. 2004. Reflex movements for a virtual human: a biology inspired approach. In SETN 2004: Proceedings of the 3rd Hellenic Conference on Artificial Intelligence, Special Session on Intelligent Virtual Environments, 525 - 534 .

Gutiérrez, M., Vexo, F., And Thalmann, D. 2005. Semantics-based representation of virtual environments. In International Journal of Computer Applications in Technology (IJCAT) Special issue - "Models and methods for representing and processing shape semantics" (to appear).

Komura, T., Ho, E. S. L., AND LAU, R. W. H. 2005. Animating reactive motion using momentum-based inverse kinematics: Motion capture and retrieval. Comput. Animat. Virtual Worlds $16,3-4,213-223$.

Leon, J.-C., Giannini, F., Falcidieno, B., Brunetti, G., Graf, H., Stork, A., Vexo, F., and Thalmann, D. 2005. Acquiring, structuring shapes and annotating them with semantics to improve design activities. In Proceedings of Virtual Concept, H. . S. aris, Berlin, Ed., 10.

MCCRAe, R. R., AND John, O. P. 1992. An introduction to the five-factor model and its applications. Journal of Personality, 60, $175-215$.
Ponder, M., Papagiannakis, G., Molet, T., MagnenatThalmann, N., And Thalmann, D. 2003. Vhd++ development framework: Towards extendible, component based vr/ar simulation engine featuring advanced virtual character technologies. cgi 00, 96.

TANG, B., PAn, Z., Zheng, L., ANd Zhang, M. 2006. Simulating reactive motions for motion capture animation. In Advances in Computer Graphics, LNCS 4035, Springer, 531-537.

Zagal, J., Mateas, M., Fernández-Vara, C., HochhalTER, B., AND LICHTI, N. 2005. Towards an ontological language for game analysis. In DIGRA Conf.

Zordan, V. B., And Hodgins, J. K. 2002. Motion capturedriven simulations that hit and react. In SCA '02: Proceedings of the 2002 ACM SIGGRAPH/Eurographics symposium on Computer animation, ACM Press, New York, NY, USA, 89-96. 